\title{
Evaluation of the biomechanical properties of the posterior eye using Shear Wave Elastography in patients with increased intracranial pressure
}

\author{
Chufan Xiao, Xiaoling Huang, Maoping Li, Jiarui Wu, Huiying Huang
}

Department of Ultrasound, the First Affiliated Hospital of Chongqing Medical University, Chongqing, China

\begin{abstract}
Aims: This study aims to investigate the biomechanical properties of the posterior eye using the shear wave elastography (SWE) in increased intracranial pressure (ICP) patients and healthy subjects. Material and methods: This study enrolled 54 healthy subjects and 54 patients with increased ICP, including 29 patients with space-occupying lesions (SOLs) and 25 patients with intracranial haemorrhage (ICH). Optic nerve sheath diameter and the stiffness of the optic nerve head, peripapillary sclera and optic nerve were evaluated. Results: Patients with increased ICP had a significantly higher optic nerve sheath diameter, Young's modulus of optic nerve head, and Young's modulus of peripapillary sclera than healthy subjects ( $p<0.01$ for all). The optic nerve sheath diameter, Young's modulus of optic nerve head and Young's modulus of the peripapillary sclera were similar between the SOLs group and ICH group ( $p>0.05$ for all). Young's modulus of the optic nerve in the ICH group was similar to the control group ( $\mathrm{p}>0.05)$, whereas the SOLs group had higher Young's modulus of optic nerve comparing the control group $(\mathrm{p}<0.05)$. Conclusion: The stiffness of the optic nerve head and peripapillary sclera were higher in patients with increased ICP than healthy subjects, and the optic nerve stiffness of SOLs patients was the highest in all subjects. The SWE could provide a quantitative analysis of the posterior eye's biomechanical properties and help the clinic diagnose the degree of visual impairments.
\end{abstract}

Keywords: optic nerve head; sclera; optic nerve; shear wave elastography; intracranial pressure

\section{Introduction}

The cranial cavity of a healthy adult is a constant and enclosed space. The volume of all components in the cranial cavity keeps constant in a dynamic balance state [1]. Increased components, such as space-occupying mass and haemorrhage in the cranial cavity, can upset the balance and lead to increased intracranial pressure (ICP). The optic nerve is surrounded by an optic nerve sheath, which is contiguous with the meninges of the brain. The optic nerve sheath's subarachnoid space directly commu-

Received 17.11.2020 Accepted 31.01.2021

Med Ultrason

2021, Vol. 23, No 3, 305-310

Corresponding author: Xiaoling Huang, MD

Department of Ultrasound, the First Affiliated Hospital of Chongqing Medical University,

1 Youyi Road, Chongqing 400016, China

Phone: 86-23-89012746

E-mail: huangxiaoling_4@163.com nicates with the intracranial subarachnoid space [2]. As a consequence of this structure, intraorbital subarachnoid space is submitted to the same pressure as the intracranial subarachnoid space [3].

Common ocular signs and symptoms of increased ICP include papilledema, optic nerve atrophy, transient obscuration, various types of visual field defects and blindness [4-6]. In patients with space-occupying lesions (SOLs), up to $50 \%$ of them present with ocular signs or symptoms [5]. In patients with intracranial haemorrhage (ICH), $55.6 \%$ of them present with papilledema and $15 \%$ present with visual disturbances [6].

In a dog model, Morgan et al found that elevation in ICP could directly impact the displacement of optic nerve head tissues [7]. Brodsky et al reported that the posterior sclera experiences change in cases with increased intracranial pressure [8]. Nusbaum et al [9] found that sustained ICP elevation resulted in retinal ganglion cell loss and optic nerve degeneration in mice. 
The posterior eye is a complex biomechanical structure. The optic nerve and peripapillary tissues, including the optic nerve head and peripapillary sclera, are critical for visual function. Biomechanical change of these tissues is thought to play an essential role in visual impairments [10]. However, research on the peripapillary tissue's biomechanical properties mainly rely on experimental animals $[7,9,11]$ and computational models $[12,13]$. The degree of optic nerve impairment is mainly reflected by the morphology parameter cup-to-disc ratio in clinical practice. Cells and tissues are sensitive to biomechanical changes [14]. However, the inaccessibility of the posterior eye has made in-vivo and quantitative analysis of its biomechanical properties challenging.

Shear Wave Elastography (SWE) is a new ultrasonographic imaging technique that provides quantitative information about tissue elasticity in real-time. Focused acoustic radiation force generated by the transducer is applied to local tissue and produces displacement inside the tissue, causing the propagation of shear waves within the selected region [15]. As the shear waves propagate, the ultrasound system tracks the shear wave speed Cs and automatically computes Young's modulus E based on the mathematical equation $\mathrm{E}=3 \rho \mathrm{Cs}^{2}$ in which $\rho$ represents tissue density [16]. The Young's modulus is directly related to the tissue's biomechanical properties [15]. The harder the tissue, the faster the speed of sound and, correspondingly, the higher Young's modulus.

This study aimed to quantitatively analyse the biomechanical properties of the posterior eye using SWE and assess its response to increased ICP insults.

\section{Material and methods}

\section{Participants}

This prospective study was conducted between May 2020 and October 2020 in the First Affiliated Hospital of Chongqing Medical University. Fifty-four healthy subjects and 54 patients with increased ICP, including 29 patients with SOLs, 25 patients with ICH were enrolled and divided into three groups: the control group, SOLs group and ICH group. The study was approved by the Ethics committee of our hospital and was designed in accordance with the Declaration of Helsinki. All participants were informed of their participation in the study and signed informed consent.

The increased ICP diagnosis was based on the invasive measurement of ICP (more than $15 \mathrm{mmHg}$ ) [17] with an inserted intraparenchymal catheter (Codman Microsensor Kit). Subjects under 18 years and those with ocular diseases (e.g., glaucoma, trauma, tumour, inflammatory disease) or with a history of ocular surgery were excluded.

\section{Ultrasonographic examination}

All ultrasound images were acquired by the Resona 7T ultrasound scanner (Mindray, Shenzhen, China) with a linear probe (L11-3U). All ultrasound images acquisitions and measurements were performed by a single explorer with 3 years of ultrasound experience. Before the study began, the explorer had received training in ultrasound elastography technique by an expert radiologist. Subjects were scanned in a supine position with eyes closed. Since the intraorbital subarachnoid spaces of the two eyes are subject to the same pressure changes, we assumed that the pathological changes of two eyes could be expected to be equivalent. One eye of each subject was selected randomly. The probe was placed perpendicularly over the eyelid through a thick layer of gel with no pressure on the eyeball.

The optic nerve was identified as a hypoechoic banded structure and surrounded by the hyperechoic optic nerve sheath. After optic nerve sheath images were recorded, the SWE was performed. During the elastography examination, grayscale and SWE images were displayed simultaneously as the dual-screen mode in real-time. The probe was held still with no pressure exerted to the eyeball until acquired stable images, then static images were stored digitally for later analysis. The motion stability (M-STB) index was applied to monitor the motion interference induced by the subject's respiration or operator's movement in real-time. M-STB index displayed on the top right corner of the screen and evaluated by stars; a reliable image should display greater than or equal to four green stars. The optic nerve sheath and elastographic images of each subject were sequentially obtained at least 3 times. All images were stored for later measurements.

The optic nerve sheath diameter was measured $3 \mathrm{~mm}$ behind the optic disc [16], and the measurement was taken between the outer edge of the hyperechoic optic nerve sheath (fig 1).

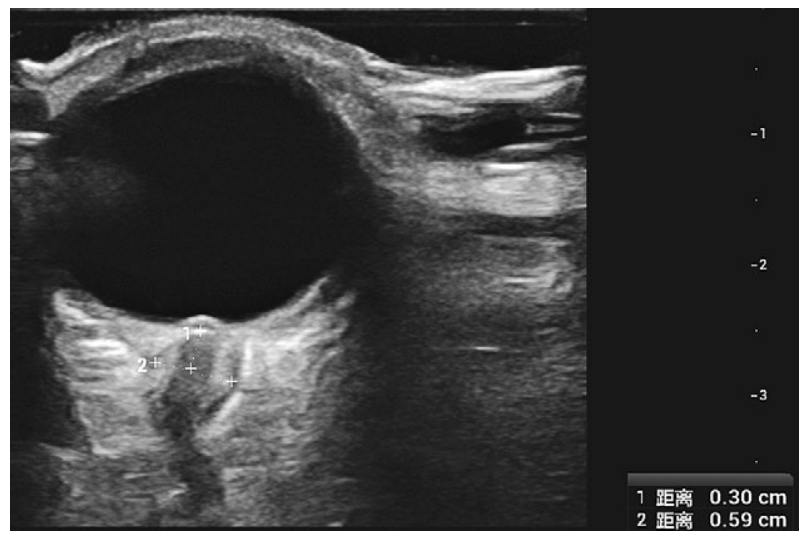

Fig 1. Measurement of optic nerve sheath diameter. 


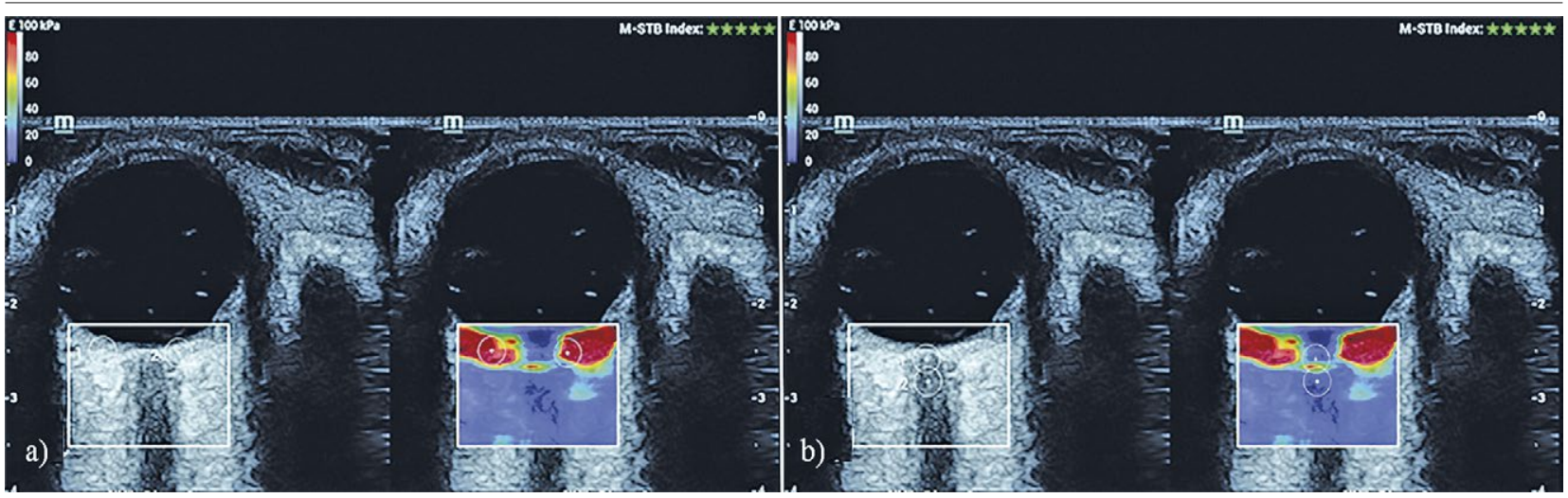

Fig 2. Shear wave elastography measurements include a) nasal sides of the peripapillary sclera (ROI 1) and temporal sides of the peripapillary sclera (ROI 2) and b) optic nerve head tissue (ROI 1) and the bulbar segment of the optic nerve (ROI 2).

SWE measurements include the nasal and temporal sides of the peripapillary sclera, optic nerve head and the bulbar segment of the optic nerve (fig 2). The size of circular regions of interest (ROI) was fixed to $3 \mathrm{~mm}$ in all measurements. The mean Young's modulus 'Emean' (kPa) within each ROI were recorded. The peripapillary sclera stiffness value was calculated as the average of the peripapillary sclera's nasal and temporal sides. The average values of three measurements were used for statistical analysis.

\section{Statistical analysis}

The normality of the distribution was assessed using the Kolmogorov-Smirnov test. Continuous variables were expressed as a mean \pm standard deviation. The independent samples $\mathrm{T}$ test, chi-square test and one-way ANOVA with a post hoc Bonferroni test were used for statistical analyses with SPSS version 23 software (SPSS Inc., Chicago, IL, United States). A p-value $<0.05$ was considered statistically significant.

\section{Results}

This study included 54 healthy subjects and 54 patients with increased ICP. The aetiologies of increased ICPs were SOLs in 29 patients (53.7\%) and ICH in 25 patients
(46.3\%). The SOLs group comprised 17 males (58.62\%) with a mean age of $47.93 \pm 10.96$ years and a mean ICP value of $23.90 \pm 4.77 \mathrm{mmHg}$. The ICH group comprised 11 males (44\%) with a mean age of $49.12 \pm 13.93$ years and a mean ICP value of $24.12 \pm 3.79 \mathrm{mmHg}$. The control group comprised 30 males $(55.56 \%)$ with a mean age of $46.94 \pm 14.16$ years. There were no significant differences between the three groups in terms of age (ANOVA, $\mathrm{p}>0.05$ ) or gender (chi-square test, $\mathrm{p}>0.05$ ). The mean ICP values between the SOLs group and ICH group were similar (independent samples t-test, $\mathrm{p}>0.05$ ).

The patients with increased ICP (the SOLs and ICH groups) had a similar optic nerve sheath diameter, Young's modulus of optic nerve head and Young's modulus of the peripapillary sclera ( $p>0.05$, for all). The control group had a significantly lower optic nerve sheath diameter, Young's modulus of optic nerve head and Young's modulus of the peripapillary sclera than the increased ICP groups ( $<<0.01$ for all). Young's modulus of the optic nerve in the ICH group was similar to the control group, whereas the SOLs group had a significantly higher Young's modulus of the optic nerve than the control group $(\mathrm{p}<0.05)$ (Table I).

In figure 3, we illustrated a SWE example from each SOLs group, ICH group, and control group.

Table I. Optic nerve sheath diameters and shear wave elastography values among groups

\begin{tabular}{llllllll}
\hline & $\begin{array}{l}\text { SOLs } \\
(\mathbf{n}=\mathbf{2 9})\end{array}$ & $\begin{array}{l}\text { ICH } \\
(\mathbf{n = 2 5})\end{array}$ & $\begin{array}{l}\text { Control } \\
(\mathbf{n = 5 4 )}\end{array}$ & p values $^{\mathbf{a}}$ & $\begin{array}{l}\mathbf{p}^{\mathbf{b}} \text { SOLs } \\
\text { vs. Control }\end{array}$ & $\begin{array}{l}\mathbf{p}^{\mathbf{b}} \text { SOLs } \\
\text { vs. ICH }\end{array}$ & $\begin{array}{l}\mathbf{p}^{\mathrm{b}} \text { ICH } \\
\text { vs. Control }\end{array}$ \\
\hline ONSD $(\mathrm{mm})$ & $5.42 \pm 0.40$ & $5.35 \pm 0.30$ & $4.54 \pm 0.28$ & $<\mathbf{0 . 0 1}$ & $<\mathbf{0 . 0 1}$ & 1.00 & $<\mathbf{0 . 0 1}$ \\
Emean of optic nerve head $(\mathrm{kPa})$ & $25.70 \pm 2.87$ & $24.79 \pm 2.96$ & $16.44 \pm 2.61$ & $<\mathbf{0 . 0 1}$ & $<\mathbf{0 . 0 1}$ & 0.69 & $<\mathbf{0 . 0 1}$ \\
Emean of PPsc $(\mathrm{kPa})$ & $82.26 \pm 8.54$ & $80.55 \pm 8.97$ & $65.97 \pm 10.69$ & $<\mathbf{0 . 0 1}$ & $<\mathbf{0 . 0 1}$ & 1.00 & $<\mathbf{0 . 0 1}$ \\
Emean of ON $(\mathrm{kPa})$ & $13.97 \pm 1.91$ & $12.97 \pm 2.26$ & $12.74 \pm 2.02$ & $\mathbf{0 . 0 3}$ & $\mathbf{0 . 0 3}$ & 0.23 & 1.00 \\
\hline
\end{tabular}

ane-way ANOVA test. ${ }^{b}$ Bonferroni post-test. Data expressed as mean \pm standard deviation. $n$, number of patients; SOLs, space occupying lesions; ICH, intracranial haemorrhage; ONSD, optic nerve sheath diameter; Emean, mean Young's modulus; ONH, optic nerve head; PPsc: peripapillary sclera; $\mathrm{ON}$, optic nerve. 


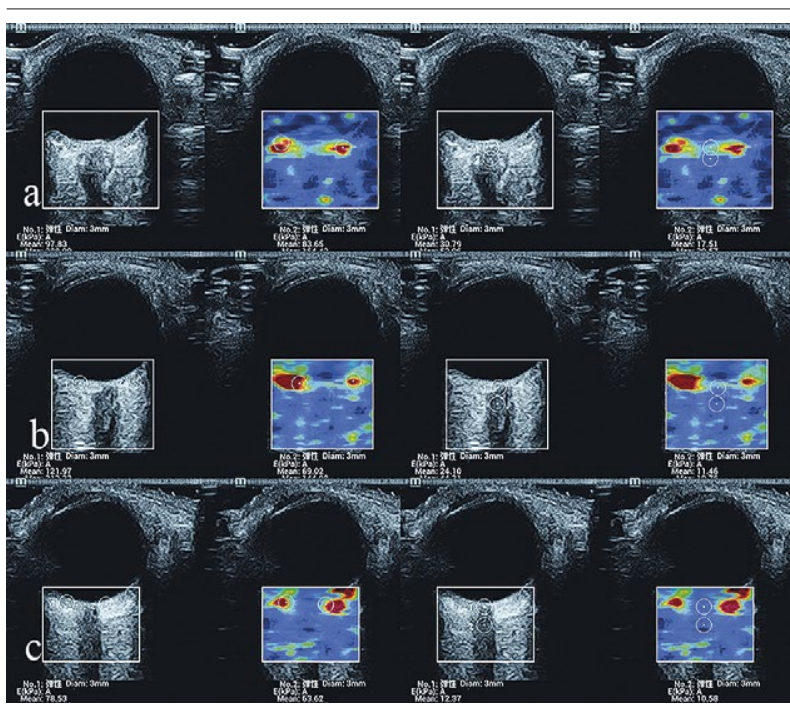

Fig 3. Shear wave elastography measurements of the posterior eye a) in a 32-year-old male with meningioma (ICP value: $29 \mathrm{mmHg}$ ), showing a mean peripapillary sclera of $90.74 \mathrm{kPa}$, optic nerve head $30.79 \mathrm{kPa}$, optic nerve $17.51 \mathrm{kPa}$; b) in a 58-year-old male with intracranial haemorrhage (ICP value: $25 \mathrm{mmHg}$ ), showing a mean peripapillary sclera of $95.50 \mathrm{kPa}$, optic nerve head $24.10 \mathrm{kPa}$, optic nerve $11.46 \mathrm{kPa}$; c) in a 46-year-old healthy male, showing a mean peripapillary sclera of $71.08 \mathrm{kPa}$, optic nerve head $12.37 \mathrm{kPa}$, optic nerve $10.58 \mathrm{kPa}$.

\section{Discussion}

The posterior eye is the most common site for pressure-related ocular diseases: In this study, SWE was applied to evaluate the biomechanical properties of the posterior eye in healthy subjects and increased ICP patients, including SOLs and ICH patients. The results demonstrated that the optic nerve head and peripapillary sclera in patients with increased ICP were stiffer than healthy subjects. Moreover, SOLs patients had higher optic nerve stiffness than other subjects.

Tissue elasticity can be an indicator of its physiological and pathological characteristics. Recent studies have applied elastography techniques to ocular tissues [18-20] and suggested that these techniques could be safely used on the human eye. There is increasing evidence that the increased ICP is involved in the visual impairment in different kinds of eye conditions $[12,21,22]$. However, elastography studies about the influence of increased ICP on the posterior eye are very limited. To date, only one published report conducted by Zhou et al [23] evaluated posterior sclera elasticity in idiopathic intracranial hypertension patients by applying ultrasound vibro-elastography. They found a higher wave speed of posterior sclera in idiopathic intracranial hypertension patients as compared to healthy subjects. To our knowledge, there have been no studies that investigate the biomechanical properties of optic nerve head and optic nerve using SWE in patients with increased ICP. To get a full understanding of biomechanical properties of the posterior eye under increased ICP, we enrolled patients with two different causes of increased ICP, including SOLs and ICH. Furthermore, we evaluated the biomechanical properties of the optic nerve head, peripapillary sclera and the optic nerve by obtaining elasticity values using SWE.

At the optic disc, the retinal ganglion cell axons converge and pass through the lamina cribrosa to form the optic nerve. The lamina cribrosa is a mesh-like connective tissue within the optic nerve head, providing structural support to the nerve fibres and blood vessels [10]. The surrounding tissues of the optic nerve head are in direct contact with the sclera [24]. The optic nerve head and peripapillary sclera are the main load-bearing areas of the globe [25]. The optic nerve is surrounded by the optic nerve sheath, which is contiguous with the meninges of the brain. Optic nerve head and the peripapillary sclera separate the intraocular space and the intracranial subarachnoid space and form a barrier between these two pressure compartments [10].

Wang et al [11] imaged the lamina cribrosa using optical coherence tomography at various intraocular pressure and ICP combinations in a monkey model and found that the lamina cribrosa deformed in response to both intraocular pressure and intracranial pressure changes. Dikici et al [18] applied SWE and found glaucoma patients with high intraocular pressure have higher optic nerve head stiffness value than healthy subjects. We found that the stiffness values of the optic nerve head in patients with increased ICP were significantly higher than healthy subjects. The possible reason for this result is that increased cerebrospinal fluid pressure disturbs the retrolaminar pressure and results in stress build-up at the optic nerve head. The optic nerve sheath's subarachnoid space is at the lower end of the cerebrospinal fluid system [26]. Increased ICP transmits to the optic nerve subarachnoidal space, causing expansion of the optic nerve sheath [27] and increased pressure in the optic nerve sheath subarachnoid [2]. The retrolaminar pressure is principally determined by the optic nerve sheath subarachnoid pressure [28]. Since the lamina cribrosa is the weak spot in the corneoscleral shell, increased retrolaminar pressure may increase stress and strain concentrations in this spot. Compression and distortion of the lamina cribrosa have direct effects of optic nerve head pathological changes [29]. According to previous studies, both acute [11] and chronic [22] elevation in ICP can lead to significant strain distribution changes within the optic nerve head.

We also found that peripapillary sclera in patients with increased ICP was significantly stiffer than healthy 
subjects, consistent with the Zhou et al study [23]. The inner one-third of the sclera constitutes the lamina cribrosa [25]. The sclera and lamina cribrosa contain a thick extracellular matrix coating; biomechanical changes in one structure can lead to entire tissue coat changes [30]. Moreover, two-thirds of the sclera is connected with the optic nerve sheath [24]; the sheath's dilation may also increase the tension acting on the peripapillary sclera.

In the elastography studies of glaucoma $[18,19]$ and multiple sclerosis [20], they found that increased optic nerve stiffness is associated with fibrosis. The SOLs are often not detected until a significant clinical symptom appears or a pronounced mass effect occurs. Mass enlarges within the enclosed cavity leads to chronic increased ICP. Increased pressure-induced deformation of the lamina cribrosa directly squeezes nerve fibres and triggers neural tissue damage. These microstructural changes within the optic nerve head can block the axoplasmic flow and eventually lead to the loss of retinal ganglion cells [25]. The central retinal artery passes through the lamina cribrosa and feeds the optic nerve and inner two-thirds of the retina. Querfurth et al [31] found reduced flow velocities and increased flow resistance of the central retinal artery in patients with chronic intracranial hypertension compare to healthy subjects. Chronic exposure to a certain amount of biomechanical insult and ischemic hypoxic injury would likely trigger nerve cell degeneration and induce neural tissue remodelling [13] in the optic nerve, consistent with the high incidence of optic nerve atrophy in SOLs patients [32]. Optic nerve remodelling accompanied by fibrosis and atrophy changes its biomechanical properties and results in increased stiffness.

However, the ICH cause is bleeding from the ruptured blood vessel due to hypertension, abnormal, or trauma. The morbidity of ICH is generally related to the acute increased of ICP. Since the optic nerve sheath has a certain degree of elasticity [25], when the ICP increases acutely, the bulbar segment of the optic nerve sheath mark expands and alleviates some of the stress on the optic nerve, which may not be enough to cause neural tissue remodelling and stiffness change. The difference in Young's modulus of the optic nerve between groups suggested that ICP may not be the only factor which influence the optic nerve stiffness. The difference in acute and chronic increased ICP may also account for the stiffness difference between groups.

There were several limitations to our study. Firstly, this study had a limited sample size; further studies with a larger sample size and longer-term follow-up assessment should be conducted. Secondly, tissue motion caused by subjects' respiration and eye movement or probe movement can affect the elasticity results. Therefore, image quality evaluation was applied, which helps minimize motion interference and ensure the SWE examination's accuracy and reproducibility. Besides, repeated measurements were obtained at least three times to reduce bias and errors.

In conclusion, we found that patients with increased ICP had a higher optic nerve head and peripapillary sclera stiffness than healthy subjects and SOLs patients had the highest optic nerve stiffness among all subjects. Elevation of ICP leads to elastic changes, firstly occurring in peripapillary structures; long-term exposure to increased pressure results in optic nerve fibrosis and increased stiffness. SWE could detect the posterior eye elasticity change in vivo and help the clinical practitioners estimate the degree of visual impairments.

Acknowledgments: This work was supported by the Graduate Student Research Innovation Project of Chongqing (grant 2019).

\section{Conflict of interest: none}

\section{Reference}

1. Smith ER, Madsen JR. Cerebral pathophysiology and critical care neurology: basic hemodynamic principles, cerebral perfusion, and intracranial pressure. Semin Pediatr Neurol 2004;11:89-104.

2. Liu D, Kahn M. Measurement and relationship of subarachnoid pressure of the optic nerve to intracranial pressures in fresh cadavers. Am J Ophthalmol 1993;116:548-556.

3. Hansen HC, Helmke K. The subarachnoid space surrounding the optic nerve. An ultrasound study of the optic nerve sheath. Surg Radiol Anat 1996;18:323-328.

4. Hayreh SS. Optic disc edema in raised intracranial pressure. VI. Associated visual disturbances and their pathogenesis. Arch Ophthalmol 1977;95:1566-1579.

5. Dermarkarian CR, Kini AT, Al Othman BA, Lee AG. Neuro-ophthalmic manifestations of intracranial malignancies. J Neuroophthalmol 2020;40:e31-e48.

6. Obuchowska I, Kochanowicz J, Mariak Z, Mariak Z. Early changes in the visual system connected with brain's aneurysm rupture. Klin Oczna 2010;112:120-123.

7. Morgan WH, Chauhan BC, Yu DY, Cringle SJ, Alder VA, House PH. Optic disc movement with variations in intraocular and cerebrospinal fluid pressure. Invest Ophthalmol Vis Sci 2002;43:3236-3242.

8. Brodsky MC. Flattening of the posterior sclera: Hypotony or elevated intracranial pressure? Am J Ophthalmol 2004;138:511.

9. Nusbaum DM, Wu SM, Frankfort BJ. Elevated intracranial pressure causes optic nerve and retinal ganglion cell degeneration in mice. Exp Eye Res 2015;136:38-44.

10. Campbell IC, Coudrillier B, Ethier CR. Biomechanics of the posterior eye: a critical role in health and disease. J Biomech Eng 2014;136:021005. 
11. Wang B, Tran H, Smith MA, et al. In-vivo effects of intraocular and intracranial pressures on the lamina cribrosa microstructure. PLoS One 2017;12:e0188302.

12. Hua Y, Tong J, Ghate D, Kedar S, Gu L. Intracranial pressure influences the behavior of the optic nerve head. J Biomech Eng 2017;139:031003.

13. Feola AJ, Myers JG, Raykin J, et al. Finite element modeling of factors influencing optic nerve head deformation due to intracranial pressure. Invest Ophthalmol Vis Sci 2016;57:1901-1911.

14. Shao Y, Tan X, Novitski R, et al. Uniaxial cell stretching device for live-cell imaging of mechanosensitive cellular functions. Rev Sci Instrum 2013;84:114304.

15. Bamber J, Cosgrove D, Dietrich CF, et al. EFSUMB guidelines and recommendations on the clinical use of ultrasound elastography. Part 1: Basic principles and technology. U1traschall Med 2013;34:169-184.

16. Ozturk A, Grajo JR, Dhyani M, Anthony BW, Samir AE. Principles of ultrasound elastography. Abdom Radiol (NY) 2018;43:773-785.

17. Albeck MJ, Børgesen SE, Gjerris F, Schmidt JF, Sørensen PS. Intracranial pressure and cerebrospinal fluid outflow conductance in healthy subjects. J Neurosurg 1991;74:597600 .

18. Dikici AS, Mihmanli I, Kilic F, et al. In vivo evaluation of the biomechanical properties of optic nerve and peripapillary structures by ultrasonic shear wave elastography in glaucoma. Iran J Radiol 2016;13:e36849.

19. Özen Ö, Özer MA, Tosun A, Özen S. Evaluation of the optic nerve and scleral-choroidal-retinal layer with ultrasound elastography in glaucoma and physiological optic nerve head cupping. Med Ultrason 2018;1:76-79.

20. İnal M, Tan S, Yumusak EM, Şahan MH, Alpua M, Örnek $\mathrm{K}$. Evaluation of the optic nerve using strain and shear wave elastography in patients with multiple sclerosis and healthy subjects. Med Ultrason 2017;19:39-44.

21. Mader TH, Gibson CR, Pass AF, et al. Optic disc edema, globe flattening, choroidal folds, and hyperopic shifts observed in astronauts after long-duration space flight. Ophthalmology 2011;118:2058-2069.
22. Villarruel JM, Li XQ, Bach-Holm D, Hamann S. Anterior lamina cribrosa surface position in idiopathic intracranial hypertension and glaucoma. Eur J Ophthalmol 2017;27:5561.

23. Zhou B, Chen JJ, Kazemi A, Sit AJ, Zhang X. An ultrasound vibro-elastography technique for assessing papilledema. Ultrasound Med Biol 2019;45:2034-2039.

24. Jonas JB, Berenshtein E, Holbach L. Anatomic relationship between lamina cribrosa, intraocular space, and cerebrospinal fluid space. Invest Ophthalmol Vis Sci 2003;44:51895195.

25. Fleischman D, Berdahl JP. Posterior scleral biomechanics and the translaminar pressure difference. Int Ophthalmol Clin 2014;541:73-94.

26. Killer HE, Laeng HR, Flammer L, Groscurth P. Architecture of arachnoid trabeculae, pillars, and septa in the subarachnoid space of the human optic nerve: anatomy and clinical considerations. Br J Ophthalmol 2003;87:777-781.

27. Amini A, Kariman H, Arhami Dolatabadi A, et al. Use of the sonographic diameter of optic nerve sheath to estimate intracranial pressure. Am J Emerg Med 2013;31:236-239.

28. Morgan WH, Yu DY, Cooper RL, Alder VA, Cringle SJ, Constable IJ. The influence of cerebrospinal fluid pressure on the lamina cribrosa tissue pressure gradient. Invest Ophthalmol Vis Sci 1995;36:1163-1172.

29. Feola AJ, Coudrillier B, Mulvihill J, et al. Deformation of the lamina cribrosa and optic nerve due to changes in cerebrospinal fluid pressure. Invest Ophthalmol Vis Sci 2017;58:2070-2078.

30. Asejczyk-Widlicka M, Pierscionek BK. The elasticity and rigidity of the outer coats of the eye. Br J Ophthalmol 2008;92:1415-1418.

31. Querfurth HW, Lagrèze WD, Hedges TR, Heggerick PA. Flow velocity and pulsatility of the ocular circulation in chronic intracranial hypertension. Acta Neurol Scand 2002; 105:431-440.

32. Tagoe NN, Essuman VA, Fordjuor G, Akpalu J, Bankah P, Ndanu T. Neuro-ophthalmic and clinical characteristics of brain tumours in a tertiary hospital in Ghana. Ghana Med J 2015;49:181-186. 